\title{
Estratégias de promoção da saúde mental na atenção primária à saúde no contexto da Covid-19: uma revisão integrativa
}

\author{
Strategies for promoting mental in primary health care in the context of Covid-19: na integrative \\ review
}

Estrategias para promover la salud mental em la atención primaria de salud em el contexto del

Covid-19: uma revisión integradora

Recebido: 17/08/2021 | Revisado: 22/08/2021 | Aceito: 25/08/2021 | Publicado: 27/08/2021

\author{
Natalia Duarte \\ ORCID: https://orcid.org/0000-0002-7612-7134 \\ Universidade Estadual do Ceará, Brasil \\ E-mail: natalia.duarte@ aluno.uece.br \\ Maria Salete Bessa Jorge \\ ORCID: https://orcid.org/0000-0001-6461-3015 \\ Universidade Estadual do Ceará, Brasil \\ E-mail: maria.salete.jorge @ gmail.com \\ Dina Mara Formiga da Silva \\ ORCID: https://orcid.org/0000-0002-5387-7194 \\ Universidade Estadual do Ceará, Brasil \\ E-mail: dinamara_silva@ hotmail.com \\ Débora Brenda Carneiro de Souza \\ ORCID: https://orcid.org/0000-0002-8503-4052 \\ Universidade Estadual do Ceará, Brasil \\ E-mail: deborabrenda@outlook.com \\ Rejane Sales Oliveira \\ ORCID: https://orcid.org/0000-0002-7284-8962 \\ Assembleia Legislativa do Ceará, Brasil \\ E-mail: rejanesales11@gmail.com \\ Paulo Barroso \\ ORCID: https://orcid.org/0000-0002-4902-1940 \\ Assembleia Legislativa do Ceará, Brasil \\ E-mail: psi.pbarrodo@gmail.com \\ Lídia Lourinho \\ ORCID: https://orcid.org/0000-0002-5883-9007 \\ Assembleia Legislativa do Ceará, Brasil \\ E-mail: lidialourinho@ hotmail.com
}

\begin{abstract}
Resumo
Objetivo: analisar o papel da Atenção Primária à Saúde (APS), e suas estratégias de promoção à saúde mental de pacientes com sofrimento psíquico na pandemia Covid-19. Método: Revisão Integrativa, orientada pela questão norteadora: Quais as estratégias de promoção à saúde mental na Atenção Primária à Saúde no contexto da Covid19, encontrados na literatura brasileira e internacional? Resultados/Discussão: Para o presente estudo foram analisados 10 artigos. A temática é abordada de diferentes formas e pontos de vista, podendo destacar-se três tópicos: O impacto da pandemia pela Covid-19 na saúde mental, o papel da APS na assistência em saúde mental e suas dificuldades no contexto da pandemia da Covid-19 e as estratégias de promoção da saúde mental na APS no contexto da pandemia do corona vírus, onde é possível observar como o sofrimento psíquico se fez presente tendo como principais origens o isolamento social, medo da morte associado ao Covid-19, violência doméstica e insegurança política e econômica, e como a tecnologia surge como importante ferramenta para promoção da saúde mental. Conclusão: Na tentativa de continuar ofertando um atendimento para a população, novas estratégias de promoção à saúde foram desenvolvidas, como a utilização da tecnologia, apesar das diferentes abordagens, ficou comprovado a eficácia dos novos métodos, e a importância de mais estudos que abordem essa temática.
\end{abstract}

Palavras-chave: Atenção primária à saúde; Covid-19; Saúde mental; Pandemia.

\section{Abstract}

Objective: analyze the role of Primary Health Care (PHC), and its strategies to promote mental health of patients with psychological distress in the Covid-19 pandemic. Method: Integrative review, guided by the guiding question: What are the strategies for promoting mental health in Primary Health Care in the context of Covid-19, found in the 
Brazilian and international literature? Results/Discussion: For the present study, 10 articles were analyzed. The theme is addressed in different ways and points of view, and three topics can be highlighted: The impact of the pandemic by Covid-19 on mental health, the role of PHC in mental health care and its difficulties in the context of the Covid-19 pandemic and the strategies for promoting mental health in PHC in the context of the corona virus pandemic, where it is possible to observe how psychic suffering was present with social isolation, fear of death associated with Covid-19, domestic violence and political and economic insecurity, and how technology emerges as an important tool for promoting mental health. Conclusion: In an attempt to continue offering care to the population, new health promotion strategies were developed, such as the use of technology, despite different approaches, the effectiveness of the new methods and the importance of further studies addressing this theme were proven.

Keywords: Primary health care; Covid-19; Mental health; Pandemic.

\section{Resumen}

Objetivo: analizar el papel de la Atención Primaria de Salud (APS) y sus estrategias para promover la salud mental de los pacientes con distrés psicológico en la pandemia Covid-19. Método: Revisión integradora, guiada por la pregunta orientadora: ¿Cuáles son las estrategias para promover la salud mental en Atención Primaria de Salud en el contexto del Covid-19, encontradas en la literatura brasileña e internacional? Resultados/Discusión: Para el presente estudio, se analizaron 10 artículos. El tema se aborda de diferentes maneras y puntos de vista, y se pueden destacar tres temas: El impacto de la pandemia de Covid-19 en la salud mental, el papel de la APS en la atención de la salud mental y sus dificultades en el contexto de la pandemia de Covid-19 y las estrategias para promover la salud mental en la APS en el contexto de la pandemia del coronavirus, donde es posible observar cómo el sufrimiento psíquico estuvo presente con el aislamiento social, el miedo a la muerte asociado con covid-19, la violencia doméstica y la inseguridad política y económica, y cómo la tecnología emerge como una herramienta importante para promover la salud mental. Conclusión: En un intento por continuar ofreciendo atención a la población, se desarrollaron nuevas estrategias de promoción de la salud, como el uso de la tecnología, a pesar de los diferentes enfoques, se demostró la efectividad de los nuevos métodos y la importancia de estudios adicionales sobre este tema.

Palabras clave: Atención primaria de salud; Covid-19; Salud mental; Pandemia.

\section{Introdução}

A Atenção Primária à Saúde (APS) é o primeiro nível de atenção em saúde e se caracteriza por um conjunto de ações que abrange a promoção e a proteção da saúde, com o objetivo de desenvolver uma atenção integral (Portela, 2017). Por tratarse da principal porta de entrada no Sistema Único de Saúde (SUS) e do centro de comunicação com toda a Rede de Atenção do SUS, funciona como um filtro capaz de organizar o fluxo dos serviços nas redes de saúde, dos mais simples aos mais complexos.

Sendo considerada a referência local de saúde, as demandas em saúde mental são muito comuns na APS, onde ocorre o primeiro contato de pacientes com transtornos mentais, principalmente com os profissionais inseridos na Estratégia de Saúde da Família (ESF). O acolhimento não corresponde somente a uma etapa inicial do atendimento, mas faz-se presente ao longo de todo o processo de atenção à saúde, implicando a responsabilidade de um acompanhamento contínuo dos usuários e suas demandas, mesmo que se verifique a necessidade de encaminhamento, que não deve resultar na perda de vínculo com a APS, com o território e com a comunidade (Lenz et al. 2021).

Esse acolhimento tornou-se essencial durante a pandemia da Covid-19, onde fez-se necessário a implementação de medidas de proteção, como o distanciamento social, uso de máscaras e reforço das medidas de higiene, tendo em vista a alta transmissibilidade do vírus e o grande número de infectados, implicando em impactos psicológicos diretamente relacionados à Covid-19, e os efeitos negativos das medidas de distanciamento social, que incluem sintomas de estresse pós-traumático, confusão, raiva, preocupações com a escassez de suprimentos, perdas financeiras e a exposição constante a notícias sobre a doença em mídias sociais, associado à redução ao acesso de recursos de apoio psicossocial como trabalho, escola, lazer, família e amigos.

Diante desse cenário, é de grande valia pontuar a importância da APS, representada pelas Unidades Básicas de Saúde (UBS) e o Núcleos de Apoio a Saúde da Família (NASF), enquanto lugar privilegiado das ações de cuidado, em um momento de emergência pública, uma vez que faz parte do itinerário de circulação frequente dos usuários com sofrimento psíquico, que 
chegam com as mais variadas demandas. Além disso, também deve-se pontuar as dificuldades que as equipes que compõem o contexto da atenção primária enfrentam em relação a falta de recursos pessoais, técnicos e estruturais para oferecer um suporte e atendimento adequado aos usuários que chegam aos serviços e estão em sofrimento psíquico, decorrente, ou não, de implicações da pandemia da Covid-19.

Tendo em vista todas essas problematizações, tem-se como objetivo analisar o papel da Atenção Primária à Saúde, e suas estratégias de promoção à saúde mental dos pacientes com sofrimento psíquico, no contexto da Covid-19.

\section{Metodologia}

Foi utilizado o método Revisão Integrativa, que tem como base a definição do tema e elaboração da pergunta de pesquisa; critérios de elegibilidade, inclusão e exclusão dos estudos; levantamento dos estudos selecionados em formato de tabelas, considerando todas as características em comum; categorização e análise crítica dos achados, identificando diferenças e conflitos; interpretação dos resultados e apresentação dos resultados, incluindo análise crítica dos achados e síntese da revisão; estágios estabelecidos por (Mendes et al., 2008; Souza et al., 2010).

Para a formulação da questão norteadora do estudo foi utilizado a pergunta PCC, a estratégia PICO adaptada, com base nos passos estabelecidos por Araújo (2020). Na adaptação, tal acrônimo significa P - população, C - conceito e C contexto/desfecho. Assim, a estratégia foi formulada da seguinte maneira: P - usuários da Atenção Primária à Saúde; C promoção à saúde mental; C - contexto da Covid-19 (Peters et al. 2015).

Desta forma, estabeleceu-se, a seguinte questão: Quais as estratégias de promoção à saúde mental na Atenção Primária à Saúde no contexto da Covid-19, encontrados na literatura brasileira e internacional?

Após identificação dos Descritores em Ciências da Saúde (DeCS), uma estratégia de busca detalhada, e individual, foi aplicada: Pubmed, LILACS via Biblioteca Virtual em Saúde (BVS), Portal Periódicos CAPES. Sendo incluído todos os artigos publicados até maio de 2021, nos últimos cinco anos. Utilizou-se três descritores extraídos do DeCS "saúde mental", "atenção primária à saúde ", "covid-19" a qual reporta significativamente ao objeto de estudo. E para a busca complementar utilizou-se sites de órgãos governamentais e a literatura cinza, utilizada com base nos achados de (Botelho, 2017).

Durante a estratégia de busca, foram elencados como critérios de inclusão: artigos completos disponíveis integralmente nas bases de dados elencadas nos idiomas português, inglês e espanhol e selecionados, artigos referentes aos últimos cinco anos, informações complementares utilizando publicações que atendessem a pergunta norteadora. Foram excluídas publicações que preenchiam pelo menos um dos seguintes critérios: (1) revisões; (2) cartas ao editor, (3) opinião pessoal de autores; (4) resumo de encontros; (5) Livros; (6) vídeos; (7) ausência de dados relacionados ao objeto e estudo.

Quadro 1 - Equações de buscas nas bases de dados.

\begin{tabular}{cl}
\hline BASE & ESTRATÉGIA DE BUSCA \\
LILACS VIA & (saúde mental) AND (atenção primária à saúde) AND (covid-19) AND ( db:("LILACS")) \\
BVS & \\
PubMed & (Mental Health) AND (Primary Health Care) AND (Coronavirus Infections) \\
Portal & (Saúde Mental) AND (Atenção Primária à Saúde) AND (Covid-19) \\
Periódicos & \\
CAPES & \\
\hline
\end{tabular}

Fonte: Autores (2021). 


\section{Resultados}

A busca resultou inicialmente em 65 artigos, tendo a seguinte distribuição entre as publicações encontradas: Portal Periódicos CAPES 19; LILACS VIA BVS 13; PUBMED 33; Sites de órgãos governamentais e serviços de saúde (n=32), totalizando 97 publicações. Em seguida, foram analisadas as publicações $(n=86)$, destes foram excluídos 87 por não se adequarem aos critérios de inclusão da pesquisa.

As etapas deste processo estão descritas na forma de um fluxograma (Figura 1).

Figura 1 - Fluxograma da seleção das publicações para a revisão integrativa baseado no modelo PRISMA.
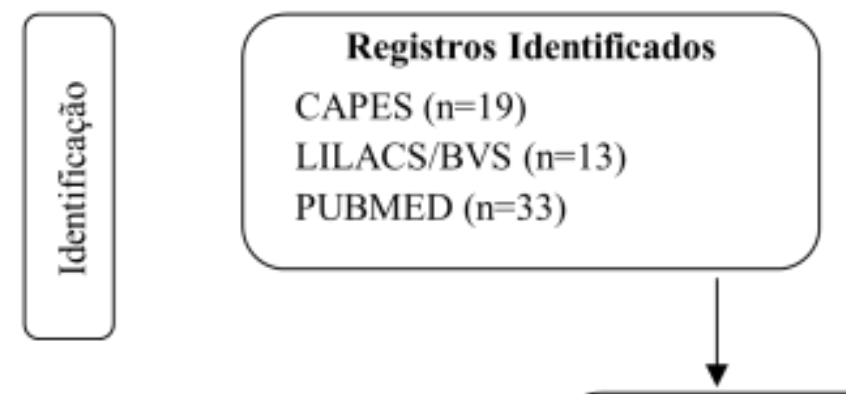

\section{Literatura cinza}

Google Scholar $(\mathrm{n}=30)$

Sites especializados $(\mathrm{n}=2)$

Total $(\mathrm{n}=32)$

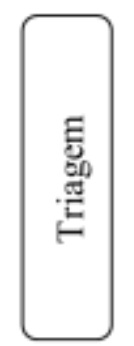

Remoção das duplicatas
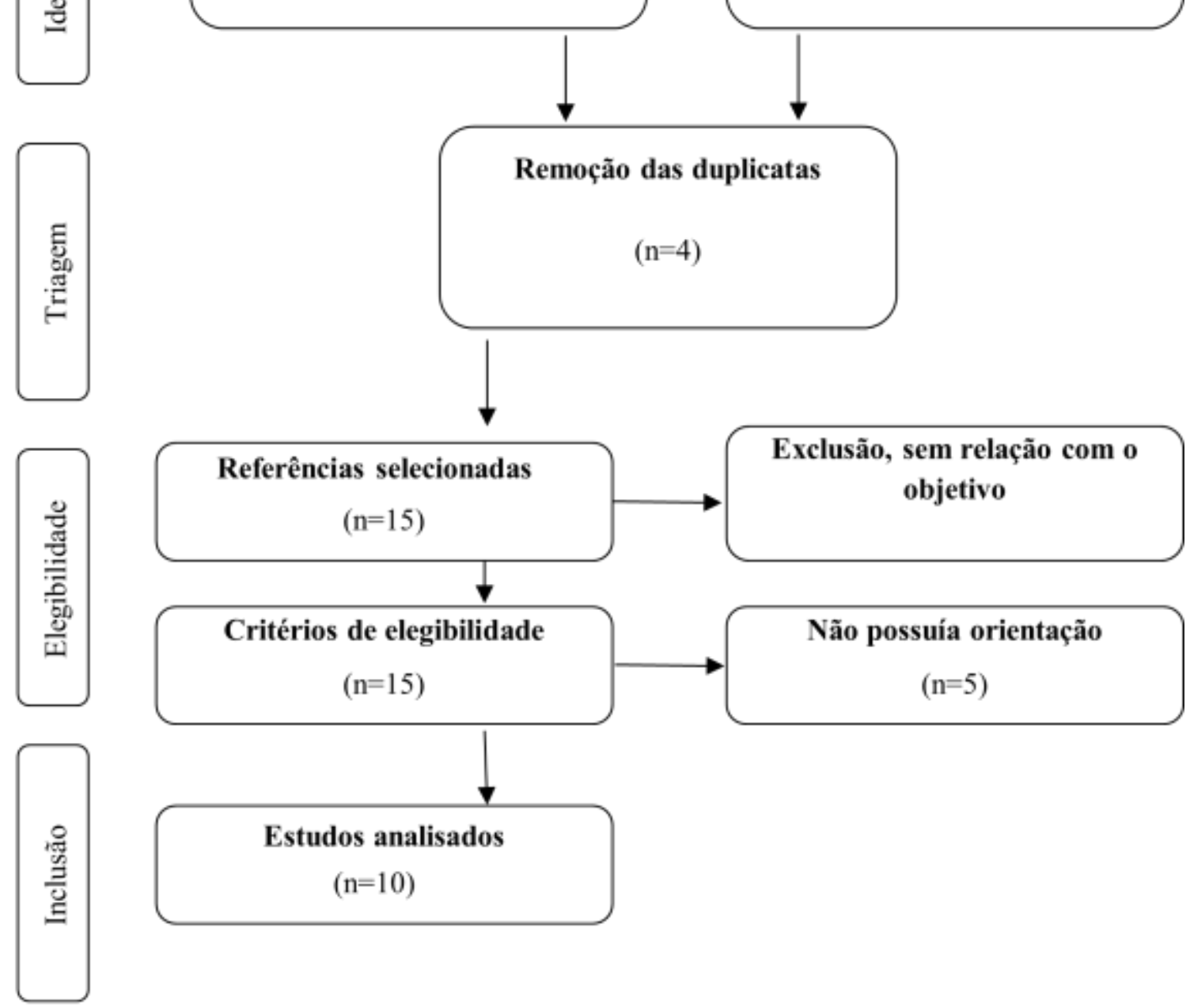

Fonte: Autores (2021).

A combinação dos termos foi selecionada e adaptada para cada base de dados (Quadro 1). O software Endnotweb foi selecionado para o manejo de todas as referências e a remoção dos artigos duplicados. Para a análise dos dados, foi utilizada a Análise de Conteúdo de Bardin (2011), estabelecendo assim categorias. 
Research, Society and Development, v. 10, n. 11, e176101119527, 2021

(CC BY 4.0) | ISSN 2525-3409 | DOI: http://dx.doi.org/10.33448/rsd-v10i11.19527

Quadro 2 - Caracterização dos estudos incluídos na Revisão Integrativa.

\section{Título}

A mobile phone-based intervention to reduce mental health problems in health care workers during the covid-19 pandemic (psycovidapp): randomized controlled trial

Covid-19 e os impactos na saúde mental: uma amostra do rio grande do sul, brasil

Effect of layperson-delivered, empathy-focused program of telephone calls on loneliness, depression, and anxiety among adults during the covid-19 pandemic: a randomized clinical trial Elaboração de vídeos para orientação de
profissionais de saúde da atenção primária no enfrentamento à pandemia da covid-19

\section{Autor}

Fiol-

DeRoque

, Et al

Periódico

Inglês/

Espanhol

JMIR Mhealth

Uhealth

Duarte, et al.

Kahlon, et al.

s, J. G. Espanhol

P., Silva, Português

E. B. S.,

Menonci

ni, C.,

Rabello,

R. S.,

Silva, S.

G.,

Borges,

Encruzilhadas da Democracia e da Saúde Mental em Tempos de Pandemia

\section{T.}

in, et al
Inglês

JAMA

Psychiatry

Revista ELO - Relato de experiência Diálogos Em

Extensão

Inglês / Ciência \& Estudo transversal

Português Saúde Coletiva quantitativo

$\begin{array}{llll}\text { Inglês/ } & \text { Psicologia: } & & \text { Qualitativa } \\ \text { Espanhol/ } & \text { Ciência } & \text { e } & \\ \text { Portuguêsl } & \text { Profissão } & \end{array}$

\section{Tipo de Publicação}

\section{Principais Resultados}

O PsyCovid App, em comparação com um aplicativo de controle, reduziu os problemas de saúde mental em 2 semanas apenas entre profissionais de saúde recebendo psicoterapia ou medicamentos psicotrópicos.

Os achados podem indicar que o distanciamento socia e a diminuição de contato físico com as pessoas durante a pandemia não é, por si só, um fator de risco para o adoecimento mental; mas sim que há influência de outros fatores que permeiam esse contexto.

Um programa de telefonema orientado para a empatia, entregue a leigos, reduziu a solidão, a depressão e a ansiedade em comparação com o grupo de controle e melhorou a saúde mental geral dos participantes em 4 semanas.

Evidenciou-se a importância de levar informações aos profissionais de

saúde da

APS sobre saúde mental e as recomendações do Ministério da Saúde sobre prevenção, proteção, manejo e notificação da COVID-19.

Identifica encruzilhadas da democracia e da saúde mental na atualidade, a exemplo da propagação de discursos antidemocráticos e do recrudescimento das fragilidades da Rede de Atenção Psicossocial. 
Research, Society and Development, v. 10, n. 11, e176101119527, 2021

(CC BY 4.0) | ISSN 2525-3409 | DOI: http://dx.doi.org/10.33448/rsd-v10i11.19527

\begin{tabular}{|c|c|c|c|c|c|}
\hline $\begin{array}{l}\text { Estratégias de cuidado à saúde mental do } \\
\text { trabalhador durante a pandemia da covid-19: uma } \\
\text { experiência na atenção primária à saúde }\end{array}$ & $\begin{array}{l}\text { Quirino, } \\
\text { et al }\end{array}$ & $\begin{array}{l}\text { Inglês / } \\
\text { Português }\end{array}$ & $\begin{array}{l}\text { Estudos } \\
\text { Universitários: } \\
\text { revista de } \\
\text { cultura }\end{array}$ & Relato de experiência & $\begin{array}{l}\text { Sinaliza a importância de novas formas de abordar a } \\
\text { saúde do trabalhador. }\end{array}$ \\
\hline $\begin{array}{l}\text { Mental health in the progress of the covid-19 } \\
\text { pandemic: conceptions of primary health care } \\
\text { workers }\end{array}$ & $\begin{array}{l}\text { Oliveira, } \\
\text { et al. }\end{array}$ & $\begin{array}{l}\text { Inglês/ } \\
\text { Espanhol/ } \\
\text { Português }\end{array}$ & $\begin{array}{l}\text { Research, } \\
\text { Society and } \\
\text { Development }\end{array}$ & Qualitativa & $\begin{array}{l}\text { Edificou-se um conjunto de implicações da pandemia } \\
\text { do novo Coronavírus } \\
\text { nas demandas de saúde mental } \\
\text { do território de atuação da Atenção Primária, } \\
\text { decorrentes da } \\
\text { s estratégias de distanciamento } \\
\text { e } \\
\text { isolamento social. }\end{array}$ \\
\hline $\begin{array}{l}\text { O impacto da pandemia pela Covid-19 na saúde } \\
\text { mental: qual é o papel } \\
\text { Da Atenção Primária à Saúde? }\end{array}$ & $\begin{array}{l}\text { Nabuco, } \\
\text { G., } \\
\text { Oliveira, } \\
\text { M. H. P. } \\
\text { P., } \\
\text { Afonso, } \\
\text { M. P. D. }\end{array}$ & $\begin{array}{l}\text { Inglês/ } \\
\text { Espanhol/ } \\
\text { Português }\end{array}$ & $\begin{array}{l}\text { Revista } \\
\text { Brasileira de } \\
\text { Medicina, de } \\
\text { Família e } \\
\text { Comunidade }\end{array}$ & Ensaio científico & $\begin{array}{l}\text { Identificou os principais fatores de risco para } \\
\text { adoecimento mental e o papel da APS na saúde mental } \\
\text { da população. }\end{array}$ \\
\hline $\begin{array}{l}\text { Pandemia de COVID-19 e a implementação de } \\
\text { teleatendimentos em saúde mental: um relato de } \\
\text { experiência na Atenção Básica. }\end{array}$ & $\begin{array}{l}\text { Araujo, } \\
\text { et al. }\end{array}$ & $\begin{array}{l}\text { Inglês / } \\
\text { Português }\end{array}$ & $\begin{array}{l}\text { Revista Saúde } \\
\text { em Redes }\end{array}$ & Relato de experiência & $\begin{array}{l}\text { Os teleatendimentos mostraram-se uma potente ação } \\
\text { terapêutica, promovendo a proteção da saúde mental } \\
\text { dos usuários e reforçando diretrizes do SUS. }\end{array}$ \\
\hline $\begin{array}{l}\text { Saúde mental em tempos de coronavírus: vídeos } \\
\text { psicoeducativos como intervenção relevante na } \\
\text { atenção primária à saúde }\end{array}$ & $\begin{array}{l}\text { Ferreira, } \\
\text { et al. }\end{array}$ & $\begin{array}{l}\text { Inglês / } \\
\text { Português }\end{array}$ & $\begin{array}{l}\text { Health } \\
\text { Residencies } \\
\text { Journal }\end{array}$ & Relato de experiência & $\begin{array}{l}\text { A utilização dos recursos midiáticos, demonstrou-se } \\
\text { adequada ao momento atual. }\end{array}$ \\
\hline
\end{tabular}

Fonte: Autores. 


\section{Discussões}

\subsection{O impacto da pandemia pela Covid-19 na saúde mental}

Tendo em vista a alta transmissibilidade do vírus, o grande número de infectados e a inexistência de medicação curativa, a recomendação global de saúde pública é baseada em medidas de distanciamento social, uso de máscaras e reforço das medidas de higiene, que apesar de serem fundamentais para desacelerar a disseminação do vírus, reduzem as interações sociais dos indivíduos, seja com a família, amigos, colegas de trabalho, faculdade ou escola (Nabuco, 2020).

Estudos com a população da China, primeiro país a adotar essas medidas preventivas, indicam que há possíveis consequências psicológicas do confinamento em massa, apresentando maior índice de ansiedade, depressão, uso nocivo de álcool e menor bem-estar mental do que os índices populacionais usuais. No entanto, o distanciamento social e a diminuição de contato físico com as pessoas durante a pandemia não é, por si só, um fator de risco para o adoecimento mental; mas sim que há influência de outros fatores que permeiam esse contexto (Duarte, 2020).

Um exemplo disso é que o ambiente e as relações existentes nele também surgem como explicativos da maior prevalência de adoecimento, levando em consideração os casos de violência doméstica, onde os principais afetados são as mulheres, idosos e crianças, abuso infantil e o abuso de substâncias lícitas e/ou ilícitas, como álcool, crack e outras drogas. (Duarte, 2020) Aliado a isso, estão as desigualdades sociais, diferenças demográficas e populações vulneráveis, têm grande impacto nas formas de exposição e de adoecimento, sendo a população mais pobre e as minorias étnicas, as mais afetadas e vítimas mortais, o que impacta diretamente na saúde mental desses indivíduos, que vivenciam a violência, medo e repressão (Dimenstein, 2020).

Além disso, essas populações também sofrem a super exposição ao vírus, que está diretamente associado às precárias condições de vida e renda, à dependência do transporte coletivo e do sistema público de saúde, que por sua vez, não está bem distribuído, nem ofertado equitativamente, à existência de doenças prévias e comorbidades que exigem continuidade de cuidados, à moradia nas periferias pobres, ao trabalho precário e informal, aos arranjos familiares com forte presença de avós como cuidadoras, facilitando a transmissão intrafamiliar e de idosos (Dimenstein, 2020).

A população que se enquadra nos grupos de risco da Covid-19, por exemplo, ser gestante, ter acima de 60 anos ou doenças preexistentes, também possuem agravantes em relação ao adoecimento mental, pois significa um maior risco de ter a doença em sua forma agravada e conhecimento das maiores taxas de mortalidade entre esses sujeitos, resultando em maior probabilidade de sofrer um impacto psicológico durante a pandemia. É importante salientar que ter um diagnóstico de transtorno mental também é considerado um fator de risco da Covid-19, pois possuem maior vulnerabilidade a infecções decorrentes do déficit no autocuidado, subestimação ou pouca atenção às medidas de prevenção, o declínio cognitivo, à frágil rede de proteção e ainda às barreiras de acesso aos serviços de saúde que usualmente estas pessoas enfrentam, contribuindo para o agravamento do quadro de saúde mental, fazendo-se necessárias intervenções específicas para esses indivíduos (Nabuco, 2020).

A existência das mídias sociais também surge como fator relevante, pois pode aproximar as pessoas através das plataformas digitais de comunicação, como chamadas por vídeo ou pedido de comida ou remédios via aplicativos, - apesar de que nem todos tem acesso a esta tecnologia por questões econômicas e sociais, além de que os idosos, de forma geral, apresentam menor familiaridade com tecnologias e dificuldade no seu manuseio - como também pode espalhar grande volume de informações que não são autênticas e nem verificadas, ora criando falsas expectativas de cura, ora provocando medo exacerbado e pânico, além da frequentemente exposição a informações sobre mortos e infectados, causando impacto negativo na saúde mental da população (Duarte, 2020; Nabuco, 2020). 
Outro fator a ser considerado são a insegurança em relação ao trabalho e à renda, sendo que aqueles que se encontram em situação ainda incerta sobre a manutenção dos seus empregos e a garantia de renda tendem a apresentar maior risco para o desenvolvimento de transtornos mentais, como estresse, ansiedade e depressão e aumento do risco de suicídios. Mas possuir um emprego garantido não é sinônimo de saúde mental, pois os trabalhadores que estão super expostos ao vírus, como os profissionais da saúde e os trabalhadores dos serviços essenciais, estão expostos a grande estresse, vivenciam tanto o medo de contrair a Covid-19 quanto de espalhar o vírus para entes queridos e pacientes saudáveis, além de fadiga, solidão, isolamento dos familiares, escassez de equipamentos de proteção individual (EPI) e aqueles expostos aos casos mais graves e mortes apresentam risco aumentado de desenvolver transtorno por estresse pós traumático (Nabuco, 2020).

Associado a tudo isto, o Brasil atravessa essa pandemia em meio a uma crise político-institucional de grandes proporções, culminando em uma instabilidade política, que é agravada pelas mudanças frequentes na liderança do Ministério da Saúde, órgão federal que deveria coordenar as ações contra a pandemia, resultando em divergências de orientações advindas de autoridades de saúde pública e governamentais, e no meio dessa disputa, a população desinformada e confusa possivelmente apresentará mais sintomas de ansiedade.

\subsection{O papel da Atenção Primária à Saúde na assistência em saúde mental e suas dificuldades no contexto da pandemia da Covid-19}

O nível primário é fundamental no planejamento de ações em saúde mental eficazes e humanizadas, que estejam de acordo com os princípios do SUS e da Reforma Psiquiátrica, visto que desenvolvem ações e atuam no cotidiano de um território geograficamente conhecido, que possibilita a construção de vínculo entre profissionais e usuários, bem como o conhecimento da realidade concreta, dos determinantes e condicionantes sociais de saúde desse espaço, merecendo destaque as equipes de Estratégia Saúde da Família, dispositivo prioritário para coordenar o cuidado e ordenar os fluxos assistenciais que delineiam os deslocamentos na rede de saúde, com vistas a garantir a integralidade da atenção e a universalização do acesso aos serviços de saúde (Martini, 2020; Nabuco, 2020; Oliveira, 2020; Quirino, 2020).

No entanto, a pandemia do corona vírus trouxe novos desafios para realização desse cuidado em saúde mental realizado pela APS, esse novo cenário exige dos serviços de atenção à saúde uma reorganização diante da necessidade de medidas de distanciamento social e de proteção, tendo em vista a alta transmissibilidade do vírus, isso resultou na restrição às consultas de rotina, individuais, e com marcação prévia, no caso dos atendimentos domiciliares, as medidas de distanciamento social adotadas pelos municípios colocaram restrições à sua realização, e as atividades de cunho educativo, como grupos e salas de espera, foram suspensas, assim como a maior parte das atividades no território (Oliveira, 2020; Quirino, 2020).

A adesão dos novos protocolos, associado à falta recursos pessoais, técnicos e estruturais para oferecer um suporte e atendimento adequado aos usuários que chegam aos serviços e estão em sofrimento psíquico, comprometeram as ações de integralidade, restringindo a assistência em saúde mental à prescrição de medicamentos, renovação de receitas, ações meramente individualizantes e medicalizantes, sem considerar outros saberes profissionais e, principalmente, a integralidade dos sujeitos, o que tira o aspecto humanizado e dinâmico, que sempre foi o diferencial positivo, da APS, fazendo-se necessário a construção de estratégias que garantam o acesso aos medicamentos, a escuta e acolhimento dos usuários durante a pandemia (Oliveira, 2020).

A pandemia causada pelo novo coronavírus deixará um rastro de ocorrência de sofrimento psíquico e transtornos psicológicos, visto que o Brasil não estava preparado para as consequências que são previsíveis no âmbito da saúde mental, na tentativa de minimizar essas consequências catastróficas,, em abril de 2020, o Ministério da Saúde do Brasil e a Organização Pan-Americana da Saúde (2020) deram início a uma campanha para promover a saúde mental no contexto da pandemia, uma 
vez que ocorreu uma eclosão de crises psiquiátricas, devido a menor adesão medicamentosa e terapêutica, especialmente, diante do fechamento de serviços de atenção psicossocial e interrupção do atendimento presencial (Dimenstein, 2020).

Oliveira (2020), traz que diante deste cenário, é de grande valia pontuar que a análise de qualquer fenômeno no campo da saúde as condições de vida das pessoas e as desigualdades sociais estão estritamente relacionadas às condições de saúde de uma população, o que ratifica a importância da Estratégia Saúde da Família enquanto lugar privilegiado das ações de cuidado, em um momento de emergência pública como este, uma vez que se fez parte do itinerário de circulação frequente dos usuários com sofrimento psíquico, que chegam com as mais variadas demandas.

\subsection{Estratégias de promoção da saúde mental na Atenção Primária à Saúde no contexto da Covid-19}

Tendo em vista os novos desafios no processo de cuidado, provocados pelas limitações físicas impostas e a necessidade de continuar a oferecer uma assistência de qualidade eficaz no âmbito da saúde mental, os profissionais de saúde que compõem as equipes multidisciplinares da APS, desenvolveram novas estratégias de intervenção e passaram a utilizar a tecnologia como nova estratégias de promoção à saúde mental da população em que estão inseridos.

Nabuco (2020), traz recomendações para garantir o cuidado de saúde mental nas comunidades em que as equipes de atenção primária atuam, como: a identificação das famílias com fatores de risco para adoecimento mental relacionados à pandemia, a articulação intersetorial para viabilizar resposta às demandas das famílias em maior vulnerabilidade, orientações à população que minimizem o adoecimento mental durante o confinamento, como procurar fontes confiáveis e oficiais de informação, manter uma rotina, respeitando o tempo de sono e horários das refeições, incluindo atividade, interagir com familiares e amigos mesmo que à distância, por telefone, mídias sociais ou videoconferência, e o apoio para minimizar as barreiras para vivência do luto daqueles que perderam entes queridos.

Ferreira (2021), traz como exemplo o uso de vídeos Psico-educativo relacionados ao enfrentamento psicológico durante a crise provocada pelo novo coronavírus. As estratégias psico-educativas são amplamente utilizadas na saúde pública como ferramenta de promoção à saúde, é um campo multidisciplinar, suas intervenções são utilizadas como meio para oferecer informações sobre um determinado fenômeno, seu desenvolvimento e suas características, seu caráter educativo e diretivo, visa incentivar o processo de conscientização sobre uma determinada realidade. Os vídeos abordam temas como: Saúde Mental Geral, Saúde mental dos profissionais de saúde, Conflitos familiares e a Violência Doméstica.

Corroborando com esse achado, Fernandes (2020) afirma que o uso de tecnologias no apoio e na divulgação de informações para os profissionais que atuam na linha de frente de combate ao coronavírus tem-se mostrado como ferramenta essencial nas práticas de educação e cuidado. A partir da experiência realizada no estudo, que também contou com a produção de vídeos, dessa vez voltado para os profissionais de saúde, evidenciou-se a importância de levar informações da APS sobre saúde mental e as recomendações do Ministério da Saúde sobre prevenção, proteção, manejo e notificação da Covid-19, pois facilitou o acesso às informações confiáveis e compactadas em vídeos, contribuindo para a boa prática de saúde mental e para atualizações a respeito do Protocolo de Manejo Clínico do Coronavírus (Covid-19) na Atenção Primária à Saúde.

Além do uso das artes visuais, a tecnologia surge com uma importante ferramenta que são as plataformas digitais de conversação online, que possibilita estratégias de cuidado por teleatendimento aos usuários. Araújo (2020), afirma que a ferramenta de teleatendimento se mostrou muito potente enquanto forma de continuar proporcionando consultas individuais e coletivas, além de diminuir idas desnecessárias à UBS, como para renovação de receitas e agendamento de exames. O teleatendimento também se mostrou eficaz no estudo de Kahlon (2021), onde ligações focadas em temas na empatia, solidão, depressão e ansiedade entre adultos foram feitas durante quatro semanas, ao final do estudo concluiu-se que houve redução da 
sensação de solidão, depressão e ansiedade em comparação com o grupo de controle e melhora na saúde mental geral dos participantes.

Já Fiol-DeRoque (2021), criou um aplicativo de comunicação que visa habilidades emocionais, comportamento de estilo de vida saudável, esgotamento e suporte social, e constatou que em profissionais de saúde que atendem pacientes com COVID-19 na Espanha, seu aplicativo, o PsyCovidApp, reduziu os problemas de saúde mental em duas semanas apenas entre profissionais de saúde recebendo psicoterapia ou medicamentos psicotrópicos.

\section{Considerações Finais}

A pandemia do novo coronavírus acarretou um impacto significativo na saúde mental da população brasileira, as estratégias de enfrentamento ao vírus, como o distanciamento social, a superexposição a notícias e informações, a instabilidade política e econômica, e a desigualdade social impactaram de forma negativa na saúde mental da população, que passou a vivenciar medo, angústia, ansiedade, depressão, violência doméstica e uso e abuso de álcool e outras drogas. Isso trouxe novos desafios para a assistência em saúde mental feita nas unidades de Atenção Primária à Saúde, que é o primeiro nível de atenção em saúde, que enfrentam a falta de recursos e infraestrutura e a perda da proximidade com a comunidade.

Na tentativa de continuar ofertando um atendimento à população, novas estratégias de promoção à saúde foram desenvolvidas, como: a utilização da tecnologia para a produção de vídeos educativos, tele consultas e desenvolvimento de aplicativos, onde, apesar das diferentes abordagens, ficou comprovado a eficácia dos novos métodos e a importância de mais estudos que abordem essa temática, visto que existe pouco material sobre o assunto e a carência de estratégias que façam a utilização da tecnologia, pois devido a desigualdade social e a instabilidade econômica, parte da população não tem acesso a internet e aparelhos eletrônicos.

Foi percebido a necessidade de mais estudos que abordem as estratégias de promoção da saúde mental na Atenção Primária, visto que é a principal porta de entrada da população no Sistema Único de Saúde, e o adoecimento mental é inevitável no contexto da pandemia da Covid-19, porém as estratégias encontradas não são integralmente aplicáveis em todos os contextos. Dessa forma, este presente trabalho visa incentivar e potencializar novas discussões sobre a temática e reflexões sobre a prática.

\section{Referências}

Araújo et al. (2020). Pandemia de COVID-19 e a implementação de teleatendimentos em saúde mental: um relato de experiência na Atenção Básica. Rev. Saúde em Redes. 6(2). https://doi.org/10.18310/2446-4813.2020v6n2\%20Suplemp\%25p

Araújo, W. C. O. (2020). Recuperação da informação em saúde: construção, modelos e estratégias. Convergências em Ciência da Informação, 3(2), 100-134. https://doi.org/10.33467/conci.v3i2.13447.

Bardin, L. (2011). Análise de conteúdo. Edições 70.

Base de dados (2021). http://www2.eca.usp.br/prof/sueli/cbd201/bases.htm.

Botelho, R. G. \& DE Oliveira, C. C. (2020). Literaturas branca e cinzenta: uma revisão conceitual. Ciência da Informação. 44(3). http://revista.ibict.br/ciinf/article/view/1804.

Dimenstein, M., Simone, A. C. R. \& Londero, M. F. P. (2020). Encruzilhadas da Democracia e da Saúde Mental em Tempos de Pandemia. Psicol. ciênc. prof. 40, e242817, 1-16. https://pesquisa.bvsalud.org/portal/resource/pt/biblio-1143509.

Duarte et al. (2020.) Covid-19 e os impactos na saúde mental: uma amostra do Rio Grande do Sul, Brasil. Ciência \& Saúde Coletiva. 25(9), 3401-3411, 2020. https://doi.org/10.1590/1413-81232020259.16472020.

EndNote Web (2021). [place unknown]; https://access.clarivate.com/login?app=endnote.

Fernandes, et al. (2020). Elaboração de vídeos para orientação de profissionais de saúde da Atenção Primária no enfrentamento à pandemia da Covid-19. Rev. 
Research, Society and Development, v. 10, n. 11, e176101119527, 2021

(CC BY 4.0) | ISSN 2525-3409 | DOI: http://dx.doi.org/10.33448/rsd-v10i11.19527

ELO - Diálogos em Extensão. 9, 1 - 8. https://doi.org/10.21284/elo.v9i.10859.

Ferreira, L. P.\& Rodrigues, M. A. F. (2021). Saúde Mental em tempos de Coronavírus: vídeos psicoeducativos como intervenção relevante na Atenção Primária à Saúde. Health Residencies Journal. 2(9). https://doi.org/10.51723/hrj.v2i9.111.

Fiol-deroque, A. et al. (2021). Mobile Phone-Based Intervention to Reduce Mental Health Problems in Health Care Workers During the COVID-19 Pandemic (PsyCovidApp): Randomized Controlled Trial. JMIR Mhealth Uhealth. https://pubmed.ncbi.nlm.nih.gov/33909587/.

Galvão T. F., Pansani, T. de S. A, \& Harrad D. (2015). Principais itens para relatar Revisões sistemáticas e Meta-análises: a recomendação PRISMA. Epidemiol e Serviços Saúde. 24 (2): 335-42. http://www.iec.pa.gov.br/template_doi_ess.php?doi=10.5123/S1679-4974201500020001 7\&scielo=S223796222015000200335.

Kahlon, et al. (2021). Effect of Layperson-Delivered, Empathy-Focused Program of Telephone Calls on Loneliness, Depression, and Anxiety Among Adults During the Covid-19 Pandemic: A Randomized Clinical Trial. JAMA Psychiatry. 78(6). https://pubmed.ncbi.nlm.nih.gov/33620417/.

Lenz, T. C, et al. (2021). Acolhimento na saúde da família - perspectivas das pessoas com deficiência que residem no rural. Rev. Enferm. UFSM. vol.11 e3: 121. https://doi.org/10.5902/2179769244155

Martini, L. C. (2020). Qual o papel da atenção primária no cuidado da saúde mental? InformaSUS-UFSCar. https://www.informasus.ufscar.br/qual-o-papel-daatencao-primaria-no-cuidado-da-saude-mental/

Mendes, K. D. S.; Silveira, R. C. C. P. \& Galvão, C. M. (2008) Revisão integrativa: método de pesquisa para a incorporação de evidências na saúde e na enfermagem. Texto Contexto Enferm, 17(4), 758-764. http://www.scielo.br/pdf/tce/v17n4/18.pdf.

Nabuco, G., Oliveira, M. H. P. P.\& Afonso, M. P. D. (2020). O impacto da pandemia pela COVID-19 na saúde mental: qual é o papel da Atenção Primária à Saúde? Rev. Brasileira de Medicina, de Família e Comunidade. 15(42). https://rbmfc.org.br/rbmfc/article/view/2532

Oliveira et al. (2020). Mental Health in the progress of the Covid-19 Pandemic: Conceptions of Primary Health Care workers. Research, Society and Development. 9(10), e9449109339. https://rsdjournal.org/index.php/rsd/article/view/9339.

Peters M. D. J., Godfrey C. M., Khalil, H., McInerney, P., Parker, D., \& Soares, C. B. (2015) Guidance for conducting systematic scoping reviews. Int J Evid Based Healthc [Internet]. 13 (3), 141-6. https://www.researchgate.net/publication/319713049_2017_Guidance_for_the_Conduct_of_JBI_Sc oping_Reviews?enrichId=rgreq 2c63bf47a03bf1c379fed09bf9a175b4-XXX\&enrichSource=Y292ZXJQYWdlOzMxOTcxMzA0OTtBUzo1N DA5MDcx MjY4OD Y0MDBA MTUw NTk3MzcxNjg4MA\%3D\%3D\&el=1_x_2\&.

Portela, G. Z. (2017). Atenção Primária à Saúde: um ensaio sobre conceitos aplicados aos estudos nacionais. Physis: Revista de Saúde Coletiva [online]. 27(2), 255-276. https://doi.org/10.1590/S0103-73312017000200005>. ISSN 1809-4481.

Quirino et al. (2020). Estratégias de cuidado à saúde mental do trabalhador durante a pandemia da covid-19: uma experiência na atenção primária à saúde. Estudos Universitários: revista de cultura. 37(1/2). https://doi.org/10.51359/2675-7354.2020.247692.

Tricco, A. C., Lillie, E., Zarin, W., O'Brien, K. K., Colquhoun, H., \& Levac D, et al. (2018). PRISMA Extension for Scoping Reviews (PRISMA-ScR): Checklist and Explanation. Ann Intern Med [Internet]. 169(7):467-73. https://www.acpjournals.org/doi/abs/10.7326/M18-0850. 